Mitteilungen der Österreichischen Geographischen Gesellschaft, 160. Jg., S. 375-383

(Annals of the Austrian Geographical Society, Vol. 160, pp. 375-383)

Wien (Vienna) 2018, https://doi.org/10.1553/moegg160s375

\title{
In Memoriam Prof. Dr. Dr. H.c. Peter Meusburger (1942-2017)
}

\author{
Werner GAMERITH, Passau*
}

mit 1 Abb. im Text

Nicht immer fallen wissenschaftliche Kapazität und menschliche Größe zusammen. Die Wissenschaftsgeschichte kennt viele hervorragende, bisweilen geniale Forscherpersönlichkeiten, die auf menschlicher Ebene jedoch - gelinde gesagt - gewöhnungsbedürftig waren, zu Exzentrik neigten, Spleens nachhingen, Bodenhaftung verloren, eine gewisse Weltfremdheit entwickelten, Schrullen kultivierten. Mit Peter Meusburger (Träger der Franz-von-Hauer-Medaille der Österreichischen Geographischen Gesellschaft von 2006) war der Geographie hingegen eine Persönlichkeit gegeben, die beides - wissenschaftliche Exzellenz und menschliche Größe - auf vortrefflichste Weise miteinander zu verbinden verstand.

Peter Meusburger, mitten in den Zweiten Weltkrieg hineingeboren, lernte bereits früh, mit Entbehrungen umzugehen. Seinen Vater, Peter Paul Meusburger, der sieben Monate vor Peters Ge-

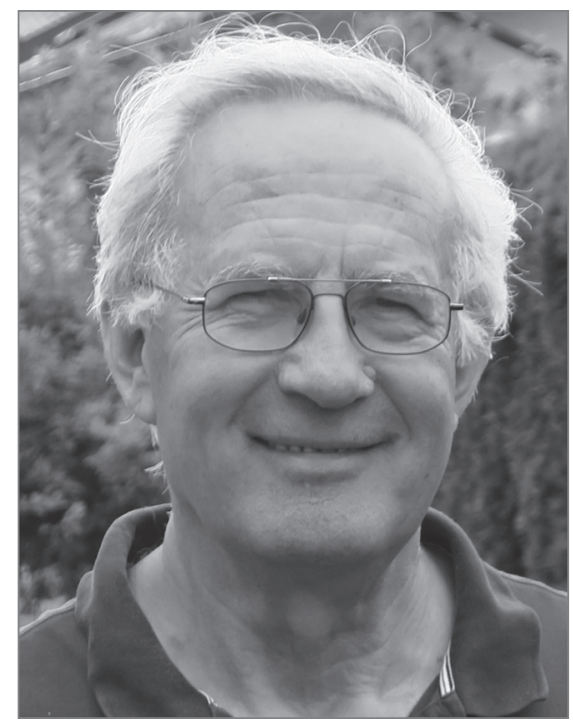

Peter Meusburger (14.03.1942-18.12.2017)

\footnotetext{
* Univ.-Prof. Dr. Werner Gamerith, Präsident der Deutschen Gesellschaft für Geographie (DGfG), Universität Passau, Fachbereich Geographie, Professur für Regionale Geographie, Innstraße 40, D-94032 Passau, Deutschland; E-Mail: Werner.Gamerith@uni-passau.de
} 
burt in Russland gefallen war, hat er nie kennenlernen dürfen. Sein Großvater, ein noch vom Ersten Weltkrieg gezeichneter Veteran, blieb die einzige männliche Bezugsperson des Einzelkinds in einem Haushalt, der stark von Frauen - tapferen Frauen - geprägt war: Peters Mutter Mina, eine unverheiratete Tante, eine verwitwete Tante und deren körperlich eingeschränkte Tochter (MEUSBURGER C. 2018). Man mag sich gut vorstellen, dass das Leben in diesen Kriegsjahren und in der unmittelbaren Nachkriegszeit trotz des dörflich-solidarischen Umfelds im vorarlbergischen Lustenau vor viele, auch materielle, Herausforderungen gestellt war.

Schon als Kind soll Peter Meusburger Weltläufigkeit gezeigt und sich für andere Kulturen interessiert haben. Als Dreijähriger entbot er einem französischen Soldaten ein „Bonjour Madame“. Er bastelte an Schiffsmodellen und besaß ein hervorragendes zeichnerisches Talent. Auch Abenteuerpläne haben den Schulbuben auf Trab gehalten - einer seiner größten Träume war es, von Lustenau aus mit einem selbstgebauten Boot den Bodensee zu erreichen (MeusBurger C. 2018). Nach einem Jahr in der Hauptschule insistierte eine Lehrerin darauf, dass Peter ans Gymnasium geschickt werden möge - sicherlich eine der wichtigsten Entscheidungen und Wendungen für sein späteres Leben: So durchlief er die Jahre am Bundesgymnasium Bregenz - sehr zur Freude seiner Mutter, die sich für ihren einzigen Sohn immer schon eine höhere Schulbildung gewünscht hatte. Tatsächlich maturierte Peter Meusburger mit ausgezeichnetem Erfolg. Dem Gymnasium folgte der Militärdienst bei den Gebirgsjägern.

1962 begann Meusburger das Studium der Geographie an der Universität Innsbruck. Auch während dieser Zeit blieb er Vorarlberg verbunden - ein Kontakt, der bei aller Weltoffenheit und Bereitschaft, sich anderen Staaten und Gesellschaften zu öffnen (schon seit den 1960er Jahren unternahm er Reisen etwa nach England, Belgien oder Osteuropa, wo er mit Dissidenten im Austausch stand), in seinem ganzen Leben niemals abriss: So widmete er sich in seinem vielbeachteten Promotionsvorhaben dem Thema der Grenzgänger in Vorarlberg. Der Dissertation lag eine akribische Datensammlung zugrunde, die sich Peter Meusburger logistisch unter Zuhilfenahme seines Mopeds erarbeitet hatte - auch in den späten 1960er Jahren waren die Zeiten einfach und die Verhältnisse bescheiden geblieben, Umstände, von denen er sich aber nie hatte entmutigen lassen und die wohl seine Gelassenheit mitgeformt haben, mit der seine Familie, seine Kollegenschaft und seine Schülerinnen und Schüler ihn später so dankbar assoziieren sollten.

Gut vorstellbar zudem, dass diese Jahre der empirischen Erhebungen entlang der österreichisch-schweizerischen Grenze seine bedingungslose Hingabe für die Belange der Wissenschaft und sein Vertrauen in die Menschen weiter zu verankern geholfen haben. Das wissenschaftliche Ergebnis dieser Studien jedenfalls konnte sich sehen lassen: 1969 wurde Peter MeusBurGER „sub auspiciis Praesidentis rei publicae“, also unter den Auspizien des Bundespräsidenten, der höchstmöglichen akademischen Auszeichnung für ein Studium, promoviert. Es spricht für seine Bescheidenheit, dass er diesen besonderen Ehrentitel, den im Schnitt weniger als ein Prozent aller österreichischen Promovenden erhält, wenn überhaupt, dann nur auf Nachfrage erwähnte. In Peter MeusBurgers umfangreichem wissenschaftlichen Euvre mit über 300 Publikationen und 30 herausgegebenen Büchern setzt die Studie zu den Grenzgängern ein gewichtiges Ausrufezeichen an markanter Position: Die Monographie „Die Vorarlberger Grenzgänger“ (Meusburger P. 1969) umfasst 618 Seiten und trägt die Nummer 1 in seinem Verzeichnis wissenschaftlicher Werke.

Eine so begabte wie integre Persönlichkeit wie Peter Meusburger für die Wissenschaft zu erhalten, bedeutete einen besonderen Gewinn für die österreichische Geographie, vor allem für das Innsbrucker Institut, an dem der eben Promovierte in den 1970er Jahren als Assistent wirkte. Zahlreiche „Testimonials“ vieler seiner Kollegen der damaligen Zeit (so etwa des Doktorvaters des Verfassers, des späteren Ordinarius für Humangeographie an der Universität Salzburg, Univ.-Prof. Dr. Helmut Heuberger) machen glaubhaft, mit welchem Fingerspitzengefühl Peter MeusBurger 
seinen Arbeitskolleginnen und -kollegen begegnete und wie sehr er mit seiner ausgleichenden Fähigkeit als ruhender Pol zum Erfolg des Instituts für Geographie der Universität Innsbruck beitrug.

1980 erhielt Peter Meusburger an der Universität Innsbruck die Lehrbefugnis für Geographie. Mit seiner Habilitationsschrift zu regionalen und sozialen Unterschieden des Ausbildungsniveaus der österreichischen Bevölkerung (Meusburger P. 1980) legte er die Basis für ein Forschungsfeld, das ihn ein Leben lang begleiten sollte und auch von vielen seiner Schülerinnen und Schüler aufgegriffen wurde. Gemeinhin wurde Peter Meusburger ab den 1980er Jahren als der Nestor der „Bildungsgeographie“ gehandelt, wobei er auch hier stets die Bescheidenheit besaß, darauf hinzuweisen, dass andere mit ihm und sogar vor ihm die Bildungsforschung in die Geographie importiert hätten. Robert GEIPEL (München) hat er in diesem Zusammenhang als Lehrer betrachtet, Alois MAYR (Münster, später Leipzig) als engen Kollegen, mit dem er in symbiotischem Austausch stand und dessen Bemühungen um einen „Nationalatlas Bundesrepublik Deutschland“ er nach Kräften unterstützte.

Drei Jahre später, 1983, folgte Peter MeusBurger einem Ruf auf den Lehrstuhl „Wirtschaftsund Sozialgeographie“ der Universität Heidelberg. Der Wechsel von Innsbruck an die älteste Universität Deutschlands (gegründet 1386) erwies sich als überaus entscheidender Schritt in seiner schon bis dahin so vielversprechenden wissenschaftlichen Biographie, denn nun stand ihm mit einem personell gut ausgestatteten Lehrstuhl die Möglichkeit offen, die Bildungsgeographie als Themenfeld wissenschaftlicher Qualifikationsarbeiten unter Mitarbeiterinnen und Mitarbeitern wie Studierenden systematischer zu platzieren und stärker zu verankern. Hätte es die Bezeichnung damals schon gegeben, wäre wahrscheinlich von einem „Thinktank“ gesprochen worden. Kohäsion und Diskussion nach innen, innerhalb des Lehrstuhls, und Kooperation und Konsens nach außen, mit den beiden anderen Heidelberger Lehrstühlen der Geographie von Dietrich BARSCH und Werner FRICKE, sicherten ein für die Geographie an der Universität Heidelberg insgesamt sehr gedeihliches und befruchtendes Arbeitsklima, das auch Bezüge zu Nachbarwissenschaften nicht nur zuließ, sondern sogar förderte - mit Kontakten zu renommierten Forscherinnen und Gelehrten an einer solcherart nicht eben armen Universität.

Der Verfasser, von 1993 bis 2004 in der glücklichen Situation, als Assistent Teil des Teams um Peter Meusburger sein zu dürfen, erinnert sich in diesem Zusammenhang etwa an die Begegnung mit dem Philosophen Hans-Georg Gadamer, mit dem Peter Meusburger bereits Kontakt geknüpft hatte und den er als besonderen Gast an einer der „Hettner Lectures“ willkommen hieß: Einer der einflussreichsten Philosophen des 20. Jahrhunderts, von allen längst als Denkmal seiner selbst geehrt und respektvoll betrachtet, kam mit Peter Meusburger scheinbar ungezwungen ins Parlieren und ließ sein betagtes Alter (geboren im Jahr 1900) vergessen machen. Es waren kurze Momente wie diese, die einen in der Erinnerung ein ganzes wissenschaftliches Leben lang begleiten werden und einem zeigten, wie wertvoll das intellektuelle Biotop war, das Peter MeUSBURGER seinen Schülerinnen und Schülern in unermüdlicher Art und mit Blick auf das Große wie das Kleine bereitete.

Ein Markenzeichen Peter Meusburgers bildete sein kontinuierliches Bemühen, Diskurse in sein Team zu tragen, Debatten anzuregen und wissenschaftliche Erkenntnisse kritisch-konstruktiv unter die Lupe zu nehmen. Zweifelsohne fühlte er sich am wohlsten, wenn er von konkret fragenden, ehrlich suchenden und offen antwortenden Menschen umgeben war; chronische Duckmäuser und permanente Ja-Sager um sich zu scharen, war seine Sache nicht. Und so schuf er viele Konstellationen, in denen die Fähigkeit geschärft werden konnte, einerseits Kritik zu formulieren und andererseits auf Kritik sachlich wie souverän zu reagieren. Fast zahllos waren die dazu einberufenen Treffen, für die er ein universitätseigenes Seminarhaus in der Abgeschiedenheit des Odenwalds („Oberflockenbach“) anmietete und zu fachlichen Debatten einlud. Das Manuskript seines wegweisenden Lehrbuchs zur Bildungsgeographie (MeusBurGER P. 1998) unterzog er auf diese Weise 
einem mehrfachen Revisionsprozess, an dem er aber mindestens so beteiligt war wie seine Umgebung. Obwohl sich dies nach dem Prinzip ,geben und nehmen“ anhört, war es sehr oft auch nur ein „geben“ seinerseits - nur zu schwer konnte man mit seinem Weitblick, seinem Wissensfundus und seiner entwaffnenden Logik mithalten.

Über die vielen Funktionen, die Peter Meusburger für die Geographie und darüber hinaus erfüllte, und die zahllosen Projekte, die er - teilweise als erster im Fach überhaupt - lancierte, wird noch zu reden sein. Wenn man diese Bilanz betrachtet, wird niemand wird umhinkommen, seine beispiellose Effizienz anzuerkennen, sein geradezu gravitätisches Arbeitsethos, seine buchstäbliche Verlässlichkeit, sein - wie es schien - perfektes Zeit-Stress-Management, das auch dort noch funktionierte, wo andere schon längst in ihren Terminen ertrunken und an ihren Verpflichtungen zerbrochen wären. Wenn eine Nachricht, völlig gegen die Regel, nicht mehr am gleichen Tag beantwortet wurde, wusste man als mit Peter MeUSBURGERs Arbeitsstil Vertrauter, dass etwas passiert sein musste oder er sich gerade in einem wichtigen Gespräch oder im Flugzeug befand. Wenig verwunderlich, dass er auch zu den pünktlichsten Menschen gehörte, die sich eine Alma Mater vorstellen und wünschen kann - und zu den innovativsten, wobei er sich selbst und den Seinen stets auch ein wenig abverlangte, zu den ersten zu zählen, die bestimmte Ideen fortentwickelten und neue Theorien zur Anwendung brachten.

Solches ließ ihn oft zur Formel „Leistung ist gleich Arbeit durch Zeit“ greifen und die Brücke zur Bildungsgeographie und seiner intensiven, auch theoretischen Beschäftigung mit Wissen schlagen, mit Plädoyers für die Bedeutung von Wissen und Wissensvorsprung, die stets auf der Seite der Sieger und der Erfolgreichen seien. Man mag dies als Überbetonung des meritokratischen Prinzips sehen, zu dem sich Peter Meusburger immer klar und unmissverständlich bekannt hat, als akademischen Drill, als Apotheose des Effizienzgedankens, doch hat er immer auch das Individuelle geachtet, hat auf persönliche Neigungen und Schwerpunkte Rücksicht genommen, hat das Menschliche im Akademischen zu Wort kommen lassen und ihm Raum gegeben. Und schließlich - das sei an dieser Stelle unterstrichen - gab der Erfolg dieser Nachwuchsschmiede Peter MEUSBURGER recht, hat doch kaum ein anderer akademischer Lehrer der gegenwärtigen deutschsprachigen Geographie so viele Personen, männlich wie weiblich, an so vielen Schlüsselpositionen der wissenschaftlichen Geographie im In- und Ausland generiert: Flensburg, Freiburg, Heidelberg, Karlsruhe, Loughborough, München, Passau.

Zeitmanagement - es gehörte zu den großen Gaben Peter Meusburgers, über die er selbst kaum sprach, geschweige denn sich als Meister der zeitlichen Einteilung und Disziplin stilisierte. Als Assistent, als der der Verfasser mit Peter Meusburger fast ein Dutzend Jahre lang eng zusammenarbeitete, stellte sich die Frage durchaus, wie er all den an ihn herangetragenen Aufgaben und sich selbst auferlegten Verpflichtungen so unbeschadet und leichtfüßig nachkommen konnte und dabei das persönliche wie das fachliche Gespräch nie zu kurz kommen ließ, wie auch ein erstaunter Gast aus Kanada, der erste aller „Hettner-Lecturer“, Derek GrEGORY, in seinem sehr feinfühligen „obituary“ zum Ausdruck brachte: „He met me at the airport in Frankfurt - a typically kind and thoughtful gesture - and we talked non-stop all the way down to Heidelberg, including a 90-minute wait while a crash on the Autobahn was cleared: we barely noticed the delay." (GREGORY 2017).

Wer nun meint, Peter Meusburger habe nichts, aber auch gar nichts außer die Wissenschaft gekannt, vergisst den Tänzer, Jazz-Liebhaber und vor allem den Familienvater. Noch in Innsbruck, 1978 und 1980, bekamen Peter und Annemarie Meusburger die beiden Töchter Catherine und Madeleine, 1984, bereits in Heidelberg, wurde die dritte Tochter, Anne-Sophie, geboren. Damit lebte Peter Meusburger erneut in einem Haushalt mit vier Frauen. Catherine Meusburger, heute Professorin am Department für Mathematik der Universität Erlangen, hält über ihren Vater als Familienmenschen fest: „Wir haben ihn als sehr liebevollen Vater in Erinnerung, der Anteil an unserem Leben nahm und sich absolut für uns einsetzte. Unsere Erziehung war von Gelassenheit, Humor 
und Großzügigkeit geprägt. Er nahm uns ernst, hatte Vertrauen in unsere Fähigkeiten und ließ uns viel Freiheit, selbstständig zu denken und Entscheidungen zu treffen." (Meusburger C. 2018). Um ehrlich zu sein: Seine Mitarbeiterinnen und Mitarbeiter wären für ihr Umfeld am Lehrstuhl zu einer ähnlichen Einschätzung gelangt, wenn man sie darauf angesprochen hätte.

In seiner wissenschaftlichen Beschäftigung mit Wissen und Qualifikation interessierte sich Peter MEUSBURGER zusehends auch für die soziologischen und sozialpsychologischen Bedingungen, unter denen sich spezifisches Wissen generiert, sich besondere Kreativität entfaltet und Innovationen entstehen. Eine Reihe wissenschaftlicher Abschlussarbeiten sind an dieser Schnittstelle zwischen Bildungs- und Wirtschaftsgeographie, Soziologie und Psychologie angefertigt worden, und so wie viele dieser Studien die Bedeutung sozialer Kontakte, persönlicher Interaktionen, wissensbasierter Netzwerke und intrinsischer Motivation für die Konstituierung kreativer Milieus unterstrichen, schuf Peter Meusburger in seinem unmittelbaren Umkreis eine Austausch- und Diskussionsplattform, auf der beständig neue Ideen gediehen. Wissenschaft als soziales System, dem ein entsprechender Nährboden bereitet werden soll - an Peter Meusburgers Lehrstuhl war dies gelebte Praxis. „Peter understood better than anyone that creative scholarship is also always irredeemably social: that it thrives on the trust that makes risk-taking possible, and on the good humour that enables genuine debate. That too was one of his most precious gifts." (Gregory 2017). Solch ein kreatives Substrat entsprang nicht einem strategischen Kalkül, sondern leitete sich aus Peter MeusBuRgers humanistischem Menschenbild und seiner Soziabilität ab, und es bereicherte nicht nur das Geographische Institut der Universität Heidelberg, sondern - durchaus und auf sehr vielfältige Art und Weise - die gesamte wissenschaftliche Geographie im deutschsprachigen Raum.

So gehen auf Peter Meusburger mehrere Initiativen zurück, die später zu einer Art „common sense" im Fach wurden, ohne dass er darauf einen Alleinvertretungsanspruch für sich reklamiert hätte. Hier ist vor allem die Institution der „Hettner Lecture“ zu nennen, ein bestechend innovatives Format, das sich den Zielen der Internationalisierung der deutschsprachigen Geographie und gleichzeitig der Förderung des wissenschaftlichen Nachwuchses verschrieb. Herausragende junge Wissenschaftlerinnen und Wissenschaftler sollten in jährlichem Turnus die Möglichkeit haben, mit höchstrenommierten Professorinnen und Professoren aus dem angloamerikanischen Raum eine Woche lang in einen intensiven Gedanken- und Meinungsaustausch einzutreten. Demgemäß wurde Heidelberg ab 1997 für ein Jahrzehnt zu einem höchst kreativen Ort des internationalen Austauschs in der Humangeographie. Über die Jahre kamen 282 Teilnehmerinnen und Teilnehmer aus 34 deutschen Universitäten und 16 weiteren Staaten zur „Hettner Lecture“ und den sie begleitenden Seminaren zusammen (GEBHARDT und GLÜCKLER 2017/18, S. 6).

Wohl keine zweite Veranstaltungsreihe hat die Humangeographie in Deutschland in den vergangenen Jahrzehnten nachhaltiger geprägt, und bemerkenswert viele der damaligen Nachwuchskräfte haben erfolgreich wissenschaftliche Karrieren beschritten und bekleiden heute Professuren. Mit den „Hettner Lectures“ wurde Heidelberg zudem zu einer Art „blue print“ für andere Geographische Institute, die ihrerseits Vortragsreihen unter internationaler Beteiligung einführten, wenngleich selten mit dem dezidierten Heidelberger Anspruch der Nachwuchsförderung und in aller Regel auch nicht mit der Intensität eines einwöchigen Gedankenaustauschs.

Möglich geworden waren die „Hettner Lectures“ nur durch Peter Meusburgers Kommunikationsgabe und seine Fähigkeit, potenzielle Mäzene zur Förderung solcher Projekte zu gewinnen in diesem Fall den Mitgründer des Softwareunternehmens SAP in Walldorf bei Heidelberg, Klaus Tschira. Aus dieser tatkräftigen Unterstützung sollte eine enge Verbundenheit des Physikers und Informatikers TsCHIRA mit dem Humangeographen MeusBurger erwachsen - und mit ihr ein Beleg dafür, welch kolossale Brücken über noch so große fachliche Distanzen der Kommunikator Peter Meusburger zu bauen verstand. Nach einer Dekade der „Hettner Lectures“ setzte er zum nächsten 
Großprojekt an, das Internationalität und Interdisziplinarität prominent verknüpfte: Mit dem Titel „Knowledge \& Space“ war die Reihe jährlicher Symposien im inspirierenden Ambiente der Villa Bosch der „Klaus Tschira Stiftung“ überschrieben, mit der Peter Meusburger sein akademisches Lebenswerk - Wissen und Bildung in räumlicher Perspektive - ab 2006 nochmals der Sicht anderer Disziplinen öffnen, kritisch zur Diskussion und konstruktiv weiterentwickeln wollte. Ein ganzes Buchregal hervorragender Synopsen auf verschiedene Facetten von Wissen und Bildung ging aus dieser wiederum großzügig von Klaus TsCHIRA geförderten Zusammenarbeit unter Beteiligung von mehr als 250 hochkarätigen Experten aus über 20 Staaten hervor (GEBHARDT und GLÜCKLER 2018, S. 33).

Wie wenige andere hat Peter MeuSBURGER die deutschsprachige Humangeographie für die internationale Bühne geöffnet und enge Kontakte in Europa und weit darüber hinaus gepflegt. Er selbst hat seine Gastprofessur (1994) an der Clark University (Worcester, Massachusetts, USA), wohin ihn seine Familie begleitete, oft als Schlüsselerlebnis bezeichnet. Die Begegnungen mit US-amerikanischen Kolleginnen und Kollegen auf wissenschaftlicher wie persönlicher Ebene hinterließen bei ihm eine tiefe Prägung, wobei er Vorzüge wie Nachteile eines Lebens in den USA dankbar und schonungslos zugleich erkannte. In der Summe mögen aber die positiven Erfahrungen überwogen haben, gemessen an der spürbaren Begeisterung, mit der er von den vielfältigen akademischen Potenzialen an den US-amerikanischen Bildungseinrichtungen (ohne die extreme Polarisierung auch im Hochschulsektor aus den Augen zu verlieren) berichtete und eine Reihe von Schülern, zu denen auch der Verfasser zählt, ermunterte, diese Chancen inhaltlich wie logistisch zu nutzen.

Bemerkenswert erscheint, dass damals, Anfang der 1990er Jahre, die deutschsprachige Humangeographie - von einigen wenigen ausgewiesenen Spezialisten abgesehen - nur sehr punktuelle und vereinzelte Beziehungen in die US-amerikanische Hochschullandschaft besaß. 1995 gehörte Peter MEUSBURGER, gemeinsam mit dem von ihm dazu gleichsam ,überredeten“ Verfasser dieser Zeilen, zu den wenigen, beinahe handverlesenen deutschsprachigen Teilnehmern des Jahreskongresses der „Association of American Geographers“ (AAG) in Chicago. In der Zwischenzeit wird diese Brücke zwischen Deutschland und den USA regelmäßig und intensiv benutzt, und Peter Meusburger gebührt auch hier der dezente Titel eines unermüdlichen und vorausblickenden Pioniers.

Auch zu Ungarn pflegte Peter Meusburger enge und vielschichtige Kontakte, die er nach der Öffnung des Eisernen Vorhangs 1989/90 weiter intensiviert hatte und mit denen er eine Reihe von Schlüsselpersonen auch außerhalb des engeren akademischen Bereichs erreichte. So war es ihm Anfang der 1990er Jahre gelungen, für detaillierte sozialräumliche Untersuchungen der ungarischen Bevölkerung große elektronische Kompendien von Originaldaten der Volkszählung zu erhalten, um diese dann in einer Serie von Abschlussarbeiten analysieren zu lassen. Nur an wenigen anderen Orten außerhalb Ungarns konnte sich damit eine so fundierte, zahlengesättigte Ungarn-Expertise etablieren wie am Geographischen Institut der Universität Heidelberg. Eine verdiente Würdigung der Ungarn-Kontakte Peter MeusBurgers erfolgte 2010 mit der Verleihung der Ehrendoktorwürde durch die Budapester Eötvös-Loránd-Universität.

Noch weiter zurück reichen Peter MeusBurgers Interesse für und seine Beziehungen zu Japan, das er nicht nur regelmäßig bereiste, sondern auch in vielen persönlichen Kontakten zu erfassen versuchte. Die dem westlichen Kulturverständnis oft so fremd erscheinende japanische Gesellschaft hat sein Interesse regelrecht beflügelt, und sein ganzes Bestreben im Austausch mit japanischen Kollegen, die in vielen von ihm initiierten Gastaufenthalten in Deutschland weilten, galt dem Verstehen der Inselkultur, der Bedeutung des Wissens in diesem Kontext und der sozialen Mechanismen. Neben Japan gehörten auch China und Brasilien zu den Großregionen, denen sich Peter MeusBurger mit besonderer wissenschaftlicher Neugier näherte und in denen er als Gastprofessor wirkte. 
Internationalität, aber auch regionale Verankerung, ob in Heidelberg oder in seiner ursprünglichen Vorarlberger Heimat - für Peter MeusBurger, einen Advokaten der internationalen Verständigung, bedeutete dies nicht notwendigerweise einen Widerspruch: Im Gegenteil, bei seltenen Gelegenheiten konnte man beobachten, wie er - der seine österreichische Staatsbürgerschaft trotz seines 34 Jahre lang währenden Lebensmittelpunkts in Heidelberg nicht aufgab - vielleicht sogar etwas verschmitzt mit seinen österreichischen Wurzeln kokettierte und um keine biographischen Anekdoten aus seiner Vorarlberger Kindheit und seinen Tiroler Studien- und Lehrjahren verlegen war. Von einer augenzwinkernden Begebenheit in diesem Zusammenhang weiß Derek GREGORY zu berichten: „As Peter pressed me for more, I recalled a remark of [Heinrich] Heine - to the effect that when the world is falling apart, one should never worry because there would always be a German professor to put it back together again - another reference to Habermas's architectonic schemes. The more I elaborated my idiotically stereotypical view of German intellectual culture, the more Peter and his colleagues roared with laughter, until eventually even I realised I was missing something; wiping away his tears, Peter managed to say 'We're all Austrian ...'” (GReGory 2017).

Auch die regelmäßigen winterlichen Ski- oder sommerlichen Wanderwochen des kompletten Lehrstuhlteams im Bregenzerwald oder im Ötztal wurzelten in dieser Tradition der Wertschätzung des Vertrauten und Familiären. Dem Land Vorarlberg blieb er zudem zeitlebens als wissenschaftlicher Beirat der Vorarlberger Landesregierung (seit 1994) verbunden; seit 2010 war er Träger des Vorarlberger Wissenschaftspreises. Seinem Lustenauer Elternhaus blieb er über die Jahrzehnte treu. Zuletzt hatte er es noch zum Altersdomizil umgebaut, sein unerwarteter Tod hat ihm jedoch den Einzug in dieses Refugium in tragisch-unerbittlicher Weise verwehrt.

Der geplante Rückzug „back to the roots“ erscheint wie ein bemerkenswerter Kontrast zum Engagement und zu den Aktivitäten, die Peter Meusburger auch nach dem Eintritt in den sogenannten Ruhestand seiner Alma Mater, der Universität Heidelberg, ebenso wie der Stadt und dem Wissenschaftsstandort Heidelberg entgegenbrachte. Seine aktive Phase als Lehrstuhlinhaber vor 2007, in der er - um hier nur einige zu nennen - die Funktionen eines Prorektors, eines Dekans, eines Senators, eines Senatssprechers und eines Rektoratsbeauftragten bekleidete, und die ebenso aktiven Jahre danach unterscheiden sich dabei nicht grundsätzlich. Wohl hatte sich Peter MeusBurger, auch aus Verantwortungsbewusstsein gegenüber den Studierenden, schrittweise von der Betreuung wissenschaftlicher Abschlussarbeiten zurückgezogen, aber als Organisator wissenschaftlicher Veranstaltungen, als „Spiritus Rector“ für gemeinschaftliche Publikationsprojekte und vor allem als Unterstützer bürgerschaftlicher Beteiligung stand er für einen ungebrochenen Tatendurst und Arbeitswillen. Das „Knowledge \& Space“-Symposion als Rückgrat dieser Jahre ist bereits genannt worden, sein vielfältiger Einsatz für die Wissenschaftsstadt Heidelberg ging jedoch noch weit darüber hinaus.

In der erstmals in der Universitätsgeschichte etablierten und seine Verdienste um die Universität sicherlich auf würdige Weise widerspiegelnden Position eines „Distinguished Senior Professor“ hat er sich als wahrer Botschafter der ehrwürdigen Ruperto Carola verstanden und ihr mit dem „Wissenschaftsatlas der Universität Heidelberg“ (2011 zur 625-Jahr-Feier der Universität erschienen; siehe MeusBuRGER und SCHUCH 2011) ein regelrecht herkulisches Denkmal gesetzt, das auch den Blick anderer Universitäten auf Heidelberg gerichtet hat. Oder, um ein Bild aus der von Peter MeusBuRGER geliebten Musik zu bedienen: Welch orchestralen Kraftakts, welch nonchalanten Dirigats bedarf es, 135 (!!) verschiedene Autorinnen und Autoren, von denen ,[d]ie überwiegende Mehrheit [...] bei ihren bisherigen Publikationen noch nie Karten verwendet [hatte]“ (Kraus et al. 2011, S. 302), zu einem so phänomenalen Erfolg zu führen, der mit einer 2012 in Englisch und 2014 in Spanisch erschienenen Ausgabe gekrönt wurde.

Peter Meusburgers Wirken für die Wissenschaft fand in den letzten Jahren immer stärker auch auf kommunaler und kommunalpolitischer Ebene Ausdruck. 2014 ließ er sich als Kandidat der 
CDU-Liste für den Heidelberger Gemeinderat aufstellen und erzielte mehr als 11.000 Stimmen. Eben dieser Gemeinderat beauftragte ihn zwei Jahre später mit einer Studie zur „Wissenschaftsstadt Heidelberg“ (MeusburGer 2016), in der er die städtebaulichen Implikationen für eine von Universität und Wissenschaft geprägte Stadt ansprach. Dabei kommt dem Neuenheimer Feld, dem wichtigsten und größten Standort der Universitäts- und Forschungseinrichtungen im Westen der Stadt, eine essenzielle Bedeutung zu, und der Bildungsgeograph Peter MeuSBURGER konnte hierzu die Forschungserfahrung eines ganzen Lebens einbringen und die Forderung erheben, dass die Stadt - wenn sie „Wissenschaftsstadt“ bleiben will - dafür auch in Zukunft räumliche Entwicklungsmöglichkeiten bereitstellen muss: Selbst bei stagnierenden Studierendenzahlen, aber steigendem Drittmittelaufkommen wächst der Druck auf die Fläche. In einer Stadt mit signifikant überdurchschnittlichen Immobilienpreisen und einer seit Langem bekannten Wohnraumknappheit ist das Plädoyer für eine weiter expandierende Wissenschaft nicht überall auf Gegenliebe gestoßen, und so hat Peter Meusburger noch in seinen letzten Monaten auch in der Kommunalpolitik und Stadtplanung lebhafte Diskussionen angestoßen, so wie er es zeitlebens als Wissenschaftler vorgelebt hat.

Nicht nur seinen Schülerinnen und Schülern und seinen Kolleginnen und Kollegen, sondern auch einer breiten Öffentlichkeit wird Peter MeusBuRGER mit der wohltuenden Kombination aus Geradlinigkeit und menschlicher Wärme in Erinnerung bleiben. Stets ist er, der so vieles für sein Fach bewegen konnte (wie etwa die Beteiligung der Geographie am „Heidelberg Center for American Studies" schon von Anbeginn dieser Initiative durch Detlef JUNKER), es in andere Institutionen und Großforschungseinrichtungen trug (so durch sein Wirken im Wissenschaftlichen Beirat des Leibniz-Instituts für Regionalentwicklung und Strukturplanung IRS in Erkner; siehe KILPER 2018), es der Öffentlichkeit erschloss (beispielsweise als Wiederbegründer 1985 und Vorsitzender der „Heidelberger Geographischen Gesellschaft“ HGG) und es bis in die obersten Verbandsgremien prägte (in seiner Funktion als Präsident der Deutschen Gesellschaft für Geographie DGfG von 2001 bis 2003), Mensch und Freund und Menschenfreund geblieben, mit einem erfrischenden Maß an Selbstironie gesegnet, von einer wohltuenden Ruhe und Gelassenheit begleitet, von unerschütterlichem Optimismus, selbst in den letzten schwersten Wochen seines Lebens, getragen („Zu Tode gefürchtet ist auch gestorben").

„He was one of the most generous, humane and modest scholars I've ever known - always keenly interested in ideas and their implications, and ever concerned to promote the work of everyone but himself." (Gregory 2017). Am 18. Dezember 2017 ist diese so eminente Stimme der Geographie nun für immer verstummt. Die Welt ohne ihn ist dunkler geworden.

\section{Literaturverweise im Text}

Gebhardt H., Glückler J. (2017/18): Nachruf: Zum Tod von Prof. Dr. Dr. h.c. Peter Meusburger. In: HGG-Journal, 32, S. 5-7.

Gebhardt H., Glückler J. (2018): Zum Tod von Prof. Dr. Dr. h.c. Peter Meusburger. In: Rundbrief Geographie, 270, S. 32-34.

Gregory D. (2017): Peter Meusburger. https://geographicalimaginations.com/2017/12/_(14.02. 2019).

KiLPER H. (2018): Das IRS trauert um Prof. Dr. Dr. h.c. Peter Meusburger. In: IRS Aktuell, 90, S. 4.

Kraus W., Lentz S., Meusburger P., Moser J., Schuch T. (2011): Wissenschaftsatlas der Universität Heidelberg - Experiment einer neuartigen Festschrift. In: Kartographische Nachrichten, 6/2011, S. 295-302.

Meusburger C. (2018): Rede zur Trauerfeier für Peter Meusburger $(* 14.03 .1942, \uparrow 18.12 .2017)$. Unveröffentlichtes Manuskript. 
Meusburger P. (1969): Die Vorarlberger Grenzgänger. Innsbruck, Kommissionsverlag (= Veröffentlichungen der Universität Innsbruck, 7 / zugleich Alpenkundliche Studien, 3).

Meusburger P. (1980): Beiträge zur Geographie des Bildungs- und Qualifikationswesens. Regionale und soziale Unterschiede des Ausbildungsniveaus der österreichischen Bevölkerung. Innsbruck, Selbstverlage des Instituts für Geographie der Universität Innsbruck (= Innsbrucker Geographische Studien, 7).

Meusburger P. (1998): Bildungsgeographie. Wissen und Ausbildung in der räumlichen Dimension. Heidelberg, Spektrum Akademischer Verlag.

Meusburger P. (2016): „Wissenschaftsstadt Heidelberg“. Analysen und Strategien. Heidelberg. https://www.heidelberg.de/site/Heidelberg_ROOT/get/params_E-1858158413/813575/ Strategie papier_Wissenschaftsstadt_2016-10-10_Dr_Peter_Meusburger.pdf(14.02.2019).

Meusburger P., Schuch T. (Hrsg.) (2011): Wissenschaftsatlas der Universität Heidelberg. 625 Jahre Ruperto Carola. Knittlingen, Bibliotheca Palatina Faksimile Verlag. 